\title{
EXTENSIONS OF TOTALLY PROJECTIVE GROUPS
}

\author{
RONALD LINTON AND CHARLES MEGIBBEN
}

\begin{abstract}
It is an unpublished observation of L. Fuchs and E. A. Walker that if $L$ is a fully invariant subgroup of the totally projective $p$-group $G$, then both $L$ and $G / L$ are totally projective. In this note we treat the more difficult converse question.
\end{abstract}

It is an unpublished observation of L. Fuchs and E. A. Walker that if $L$ is a fully invariant subgroup of the totally projective $p$-group $G$, then both $L$ and $G / L$ are totally projective. In this note we treat the more difficult converse question.

Recall that a reduced abelian $p$-group $G$ is said to be totally projective if $p^{\alpha} \operatorname{Ext}\left(G / p^{\alpha} G, K\right)=0$ for all abelian groups $K$ and all ordinals $\alpha$. There are numerous characterizations of totally projective groups more enlightening than the definition [1, p. 90], but the main fact that we need is the following observation: If $G$ is totally projective, then so are $p^{\alpha} G$ and $G / p^{\alpha} G$ for all ordinals $\alpha$; and, conversely, if there is an ordinal $\alpha$ such that both $p^{\alpha} G$ and $G / p^{\alpha} G$ are totally projective, then $G$ is totally projective. It is, of course, this latter statement which we shall generalize.

We shall also require at a crucial juncture another characterization of totally projectives which may be viewed as a generalization of the classical Kulikov criterion. For this we need certain definitions. If $\lambda$ is a limit ordinal, we call a $p$-group $G$ a $C_{\lambda}$-group provided $G / p^{\alpha} G$ is totally projective for all $\alpha<\lambda$. Thus every abelian $p$-group is a $C_{\omega}$-group. Recall that the length of a reduced $p$-group $G$ is just the smallest ordinal $\lambda$ such that $p^{\lambda} G=0$. We shall say that a reduced abelian $p$-group $G$ is $\sigma$-summable if its socle $G[p]=\{x \in$ $G: p x=0\}$ is the ascending union of a sequence of subgroups $\left\{S_{n}\right\}_{n<\omega}$ where for each $n$ there is an ordinal $\alpha_{n}$ less than the length of $G$ such that $S_{n} \cap p^{\alpha_{n}} G=0$. We can now state

THEOREM 1. Let $\lambda$ be a limit ordinal cofinal with $\omega$. Then a p-group $G$ of length $\lambda$ is totally projective if and only if $G$ is a $\sigma$-summable $C_{\lambda}$-group.

Proof. Let $G$ be a totally projective group having limit length $\lambda$ cofinal with $\omega$. Then $G / p^{\alpha} G$ is totally projective for all $\alpha$ and thus $G$ is a $C_{\lambda}$-group. Since $G$ has limit length, it is a direct sum of groups of length strictly less than $\lambda[1$, p. 97]. As $\lambda$ is cofinal with $\omega$, we actually have such a direct decomposition of $G$ into countably many summands $G=\bigoplus_{n<\omega} H_{n}$ where we may take

Received by the editors January 21, 1976.

AMS (MOS) subject classifications (1970). Primary 20K 10.

Key words and phrases. Totally projective group, fully invariant subgroup, $\mathrm{C}_{\lambda}$-groups.

(1) American Mathematical Society 1977 
$\alpha_{n}=$ length of $H_{n}$ strictly less than $\lambda$ and $\alpha_{n}<\alpha_{n+1}$. Taking $S_{n}$ to be the socle of $H_{0}+\cdots+H_{n}$, we see that $G$ is $\sigma$-summable.

Conversely, suppose $G$ is a $\sigma$-summable $C_{\lambda}$-group of length $\lambda$. There are two distinct cases. First if $\lambda=\beta+\omega$ for some ordinal $\beta$, then the $\sigma$-summability of $G$ implies that $\left(p^{\beta} G\right)[p]$ is the ascending union of subgroups of bounded height (as computed in $p^{\beta} G$ ) and, hence, $p^{\beta} G$ is a direct sum of cyclic groups by the classical Kulikov criterion. But then we have both $p^{\beta} G$ and $G / p^{\beta} G$ totally projective and, therefore, $G$ is totally projective.

Finally, assume that $\lambda \neq \beta+\omega$ for all $\beta$ and let the $S_{n}$ 's and $\alpha_{n}$ 's be as in the definition of $\sigma$-summability. Since $\alpha_{n}+\omega<\lambda$ for all $n$, we may assume that each $\alpha_{n}$ is of the form $\beta_{n}+\omega$. Then by a routine argument we can enlarge each $S_{n}$ so that it is maximal disjoint in $G[p]$ from $p^{\alpha_{n}} G$ and still maintain the monotonicity of the sequence $\left\{S_{n}\right\}_{n<\omega}$. Indeed such an argument occurs in the standard proofs of the Kulikov criterion. Next we form an ascending sequence of subgroups $\left\{G_{n}\right\}_{n<\omega}$ where $G_{n}$ is maximal in $G$ with respect to having $S_{n}$ as its socle. Then $G=\cup_{n<\omega} G_{n}$ is totally projective by [3, Proposition 2.5].

Recall that the height $h_{G}(x)$ of a nonzero element $x$ in the reduced $p$-group $G$ is defined to be the least ordinal $\alpha$ such that $x \notin p^{\alpha+1} G$. We also set $h_{G}(0)=\infty$. Let $\alpha=\left(\alpha_{i}\right)_{i<\omega}$ be an increasing sequence of ordinals and symbols $\infty$; that is, for each $i$, either $\alpha_{i}$ is an ordinal or $\alpha_{i}=\infty$ and $\alpha_{i}<\alpha_{i+1}$ provided $\alpha_{i} \neq \infty$. With each such sequence $\alpha$ we associate the fully invariant subgroup $G(\boldsymbol{\alpha})$ of the $p$-group $G$ defined by

$$
G(\boldsymbol{\alpha})=\left\{x \in G: h_{G}\left(p^{i} x\right) \geqslant \alpha_{i} \text { for all } i<\omega\right\} .
$$

If $G$ is totally projective, then all of its fully invariant subgroups are of this form (see [1, Theorem 67.1 and Exercise 6, p. 101]). Thus we shall satisfy ourselves with showing that if for some sequence $\boldsymbol{\alpha}$ both $G(\boldsymbol{\alpha})$ and $G / G(\boldsymbol{\alpha})$ are totally projective, then $G$ itself is necessarily totally projective. We suspect that there does not exist a $p$-primary group $G$, necessarily not totally projective, that contains a fully invariant subgroup $L$ not of the form $G(\alpha)$ such that both $L$ and $G / L$ are totally projective.

Our first result will turn out to be a major step towards proving the desired theorem.

THEOREM 2. Let $\lambda$ be a limit ordinal cofinal with $\omega$ and let $\boldsymbol{\alpha}=\left\{\alpha_{i}\right\}_{i<\omega}$ be an increasing sequence of ordinals bounded above by $\lambda$. If $G$ is a $C_{\lambda}$-group and if $G(\boldsymbol{\alpha})$ is totally projective, then $G$ is totally projective.

Proof. Let $L=G(\boldsymbol{\alpha})$. First we wish to show that it is enough to prove the theorem in the special case where (1) $\lambda=\sup \alpha_{i}$, (2) $\lambda$ is the length of $G$, and (3) $L$ is a direct sum of cyclic groups. Let $\delta=\sup \alpha_{i} \leqslant \lambda$. It is not difficult to verify that $p^{\omega} L=p^{\delta} G$ and, hence, $p^{\delta} G$ is totally projective. Therefore we need only establish that $G / p^{\delta} G$ is totally projective. But if $\delta<\lambda$, this follows from the assumption that $G$ is a $C_{\lambda}$-group. Hence we may assume that $\lambda=\delta=$ $\sup \alpha_{i}$. Then it is easily seen that $L / p^{\omega} L=L / p^{\lambda} G=\left(G / p^{\lambda} G\right)(\boldsymbol{\alpha})$ and, of 
course, $L / p^{\omega} L$ is a direct sum of cyclic groups since $L$ is totally projective. Hence the desired reduction follows since $G / p^{\lambda} G$ is a $C_{\lambda}$-group if $G$ is.

Since now we are assuming $L$ to be a direct sum of cyclic groups, we have $L[p]=\cup_{n=1}^{\infty} T_{n}$ where $T_{n} \cap p^{n} L=0$ and $T_{n} \subseteq T_{n+1}$ for each $n$. Now clearly $L[p]=\left(p^{\alpha_{\alpha}} G\right)[p]$ and, hence, we have a direct decomposition $G[p]=S_{0}+$ $L[p]$ where $S_{0} \cap p^{\alpha_{0}} G=0$. It follows that $G[p]$ is the monotone union of the sequence of subgroups $\left\{S_{n}\right\}_{n<\omega}$ where $S_{n}=S_{0}+T_{n}$ for $n \geqslant 1$. Moreover, $S_{n} \cap p^{\alpha_{n}} G=0$ for each $n$ since

$$
S_{n} \cap p^{\alpha_{n}} G=S_{n} \cap\left(p^{\alpha_{n}} G\right)[p]=S_{n} \cap\left(p^{n} L\right)[p]=S_{n} \cap p^{n} L=0 .
$$

We conclude that $G$ is $\sigma$-summable and, therefore, Theorem 1 is applicable.

Next we turn to the very special case of our problem where $\alpha_{0}=\alpha$ and $\alpha_{i}=\infty$ for $i>1$.

Lemma. If $L=\left(p^{\alpha} G\right)[p]$ and $G / L$ is totally projective, then $G$ is totally projective.

Proof. First note that

$$
p^{\alpha+1} G=p\left(p^{\alpha} G\right) \cong\left(p^{\alpha} G\right) /\left(p^{\alpha} G\right)[p]=p^{\alpha}(G / L)
$$

and, hence, $p^{\alpha+1} G$ is totally projective. But this clearly implies that $p^{\alpha} G$ is totally projective, and it remains only to show that $G / p^{\alpha} G$ is also totally projective. Since $L \subseteq p^{\alpha} G$, we have $G / p^{\alpha} G \cong(G / L) / p^{\alpha}(G / L)$, and the latter group is totally projective since $G / L$ is.

Corollary. If $L=G(\boldsymbol{\alpha})$ and $G / L$ is totally projective, then $G / p L$ is totally projective.

Proof. Observe that $L / p L=p^{\alpha}(G / p L)[p]$ where $\alpha=\alpha_{0}$. Then $(G / p L) /(L / p L) \cong G / L$ is totally projective and we need only apply the lemma to $G / p L$.

We are now in position to prove

THEOREM 3. If $\boldsymbol{\alpha}$ is an increasing sequence of ordinals and symbols $\infty$ such that both $G(\boldsymbol{\alpha})$ and $G / G(\boldsymbol{\alpha})$ are totally projective p-groups, then $G$ itself is totally projective.

Proof. Let $L=G(\boldsymbol{\alpha})$. If the sequence $\boldsymbol{\alpha}$ contains any symbols $\infty$, then there is a positive integer $n$ such that $p^{n} L=0$. But then repeated applications of the above corollary yield the desired conclusion that $G$ is totally projective. Thus we may assume that $\boldsymbol{\alpha}=\left\{\alpha_{i}\right\}_{i<\omega}$ is an increasing sequence of ordinals and take $\lambda=\sup \alpha_{i}$. By Theorem 2, it suffices to show that $G$ is a $C_{\lambda}$-group. Hence we need only verify that $G / p^{\alpha_{n}} G$ is totally projective for all $n$. But since $p^{n} L \subseteq p^{\alpha_{n}} G$, we have

$$
G / p^{\alpha_{n}} G \cong\left(G / p^{n} L\right) / p^{\alpha_{n}}\left(G / p^{n} L\right)
$$

totally projective since each $G / p^{n} L$ is totally projective by our Corollary above. 


\section{REFERENCES}

1. L. Fuchs, Infinite Abelian groups, Vol. II, Academic Press, New York, 1973.

2. C. K. Megibben, The generalized Kulikov criterion, Canad. J. Math. 21 (1969), 1192-1205. MR 40 \#2754.

3. K. D. Wallace, $C_{\lambda}$-groups and $\lambda$-basic subgroups, Pacific J. Math. 43 (1972), 799-809. MR 47 \#3569.

Department of Mathematics, University of South Alabama, Mobile, Alabama 36688

Department of Mathematics, Vanderbilt University, Nashville, TenNessee 37235 